hep-lat/0210008

RBRC-252

\title{
Nucleon Decay Matrix Elements for Domain-Wall Fermions
}

\author{
Yasumichi Aoki ${ }^{\mathrm{a}}[\mathrm{RBC}$ collaboration] * \\ ${ }^{a}$ RIKEN BNL Research Center, Brookhaven National Laboratory, Upton, NY 11973, USA
}

\begin{abstract}
We report on the nucleon decay matrix elements with domain-wall fermions in quenched approximation. Results from direct and indirect method are compared with a focus on the process of a proton decaying to a pion and a lepton. We discuss the renormalization necessary for the matching to the continuum theory. Preliminary results for the renormalized chiral lagrangian parameters are presented.
\end{abstract}

\section{INTRODUCTION}

Nucleon decay is one of the most important aspect that any (SUSY) GUT model has. In low-energy effective theories it is represented by dimension six operators made of one lepton field and three quark fields. In the decay of $p \rightarrow \pi^{0}+e^{+}$, for example, we need to calculate hadronic matrix element of the three quark operator $\mathcal{O}_{R / L ; L}^{\not B}=\epsilon^{i j k}\left(u^{i T} C P_{R / L} d^{j}\right) P_{L} u^{k}$ with initial proton and final pion states, where $C$ is the charge conjugation matrix. $P_{R / L}$ represents the right or left projection matrix, respectively.

Lattice calculation of the nucleon decay matrix elements historically started with the indirect calculation 11. First, one estimates the overlap of the operator to the proton state, $\alpha$ and $\beta$,

$\begin{aligned} \alpha P_{L} u_{p} & \equiv\left\langle 0\left|\mathcal{O}_{R ; L}^{\not B}\right| p ; \vec{k}=\overrightarrow{0}\right\rangle \\ \beta P_{L} u_{p} & \equiv\left\langle 0\left|\mathcal{O}_{L ; L}^{\not B}\right| p ; \vec{k}=\overrightarrow{0}\right\rangle\end{aligned}$

with $u_{p}$ being the proton spinor. Then, with the help of chiral perturbation theory, one can calculate the nucleon decay matrix elements. The first attempt to calculate the nucleon decay matrix element directly handling the three point function is done by Gavela et. al [2], showing significant deviation from the indirect calculations. Recently JLQCD [3] pointed out the incompleteness of the calculation [2]. The matrix element has a tensor structure,

$$
\left\langle\pi ; \vec{p}\left|\mathcal{O}_{R / L ; L}^{\not B}\right| p ; \vec{k}\right\rangle=P_{L}\left[W_{0}-i \not q W_{q}\right] u_{p},
$$

\footnotetext{
*We thank RIKEN, Brookhaven National Laboratory and the U.S. Department of Energy for providing the facilities essential for the completion of this work.
}

where $q=k-p$. The relevant form factor $W_{0}$ is what we need since the term proportional to $\not q$ vanishes after multiplying the lepton spinor. $W_{q}$ is called the irrelevant form factor. JLQCD uses quenched Wilson gauge configurations and Wilson fermion at parameters summarized in Table 1 (c). The three quark operators are renormalized with one-loop perturbation theory. Now the difference of direct and indirect calculations is not huge, but, still 30 to $40 \%$ for most cases.

In this study we try to calculate nucleon decay matrix elements with domain-wall fermions in the quenched approximation. Using DBW2 gauge action makes chiral symmetry breaking especially small compared to the other actions [4,5]. With this good chiral symmetry one expects the good property to calculate hadronic matrix elements: 1) preventing operator mixing with different chiral structure, and 2) good scaling, even down to $1 / a \simeq 1 \mathrm{GeV}$ region [6,5]. We restrict ourselves to the case of degenerate quark mass in the meson. In this case, one can calculate $p \rightarrow \pi^{0}+l^{+}$ and $p \rightarrow \pi^{+}+\bar{\nu}$ decay amplitudes. The latter is obtained by multiplying the former by $\sqrt{2}$ under the exact $\mathrm{SU}(2)$ symmetry of $u$ and $d$ quarks.

\section{PROCEDURE AND RESULTS}

The parameters of our simulation are shown in Table 1 (a). Our quark propagator is obtained by averaging two propagators calculated using periodic and anti-periodic boundary conditions. Thus, the effective temporal extent is 64. 100 independent gauge configurations are analyzed for the three and two point functions to calculate the 
Table 1

Lattice parameters and corresponding inverse lattice spacings with $\rho$ mass input. Values of $r_{0}$ are listed for another estimate of the scale. We use the parameter set (a) for the nucleon decay. $\dagger$ and $\ddagger$ represent unpublished data with 50 and 100 configurations respectively.

\begin{tabular}{ccccccccccc}
\hline & fermion & size $(V \times T)$ & $L_{s}$ & $M_{5}$ & $1 / a_{\rho}[\mathrm{GeV}]$ & ref. & gauge & $6 / g^{2}$ & $r_{0} / a$ & ref. \\
\hline (a) & DWF & $16^{3} \times 32$ & $\mathbf{1 2}$ & $\mathbf{1 . 8}$ & $1.23(5)$ & $\dagger$ & DBW2 & $\mathbf{0 . 8 7}$ & \multirow{2}{*}{$3.58(4)$} & $\ddagger$ \\
(b) & DWF & $16^{3} \times 32$ & 16 & 1.8 & $1.31(4)$ & {$[5$} & DBW2 & 0.87 & & \\
\hline (c) & Wilson & $28^{3} \times 48 \times 80$ & - & - & $2.30(4)$ & {$[3$} & Wilson & 6.0 & \multirow{2}{*}{5.368} & 河 \\
(d) & DWF & $16^{3} \times 32$ & 16 & 1.8 & $1.92(4)$ & {$[6]$} & Wilson & 6.0 & & \\
\hline
\end{tabular}

ratio [3],

$R(t)=\frac{\left\langle J_{\pi}\left(t_{1}\right) \mathcal{O}_{R / L ; L}^{B}(t) \bar{J}_{p}\left(t_{0}\right)\right\rangle}{\left\langle J_{\pi}\left(t_{1}\right) J_{\pi}^{\dagger}(t)\right\rangle\left\langle J_{p}(t) \bar{J}_{p}\left(t_{0}\right)\right.} \sqrt{Z_{\pi} Z_{p}}$.

In the three point function proton and pion interpolating fields are located at $t_{0}=6$ and $t_{1}=24$ respectively. Momentum $\pm \vec{p}$ with $\vec{p} a=$ $(1,0,0) \pi / 8$ or $(1,1,0) \pi / 8$ is injected to the pion and the operator in the three point function, as well as in the pion two point function in the denominator. $\sqrt{Z_{\pi}}$ and $\sqrt{Z_{p}}$ are overlap of $J_{\pi}$ and $J_{p}$ to the corresponding pion and proton states, which is estimated from the fit of two point functions. We take four different quark masses $m_{f} a=0.02,0.04,0.06,0.08$, among which the strange mass is located between the last two. After appropriate projection, trace and the subtraction of the irrelevant contribution, $W_{0}$ is calculated with a fit to plateau of the ratio. The results with bare operator $\mathcal{O}_{L ; L}^{\not B}$ are shown in Figure 1. $W_{0}$ can only depend on $q^{2}$ and on $m_{f}$ which can enter through the masses of proton and pion. We assume a form of the fitting function of $W_{0}=c_{0}+c_{1} q^{2}+c_{2}\left(q^{2}\right)^{2}+c_{3} m_{f}$. In our precision the physical kinematics are approximated as $m_{f}=0$ and $-q^{2}=0$. The filled diamond in the figure represents the result extrapolated to this point. We also calculate the chiral lagrangian parameters $\alpha$ and $\beta$ extrapolated to $m_{f}=0$. By the tree-level chiral perturbation theory [8], the relevant part of the matrix element is obtained,

$\left\langle\pi^{0}\left|\mathcal{O}_{L ; L}^{\not B}\right| p\right\rangle_{\mathrm{rel}} \simeq \beta(1+D+F) P_{L} u_{p} / \sqrt{2} f$,

where $D=0.47$ and $F=0.80$ from the experiment [9], and $f$ is $f_{\pi}=0.131 \mathrm{GeV}$. The open

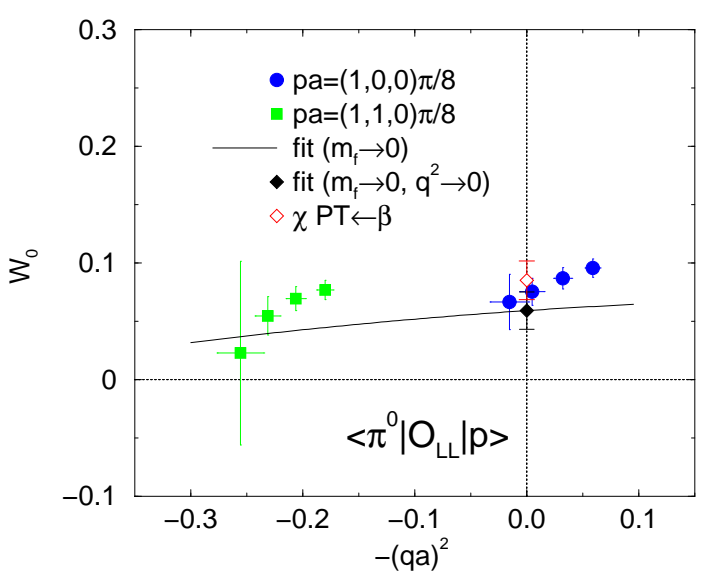

Figure 1. $W_{0}$ for $p \rightarrow\left(\pi^{0}, l^{+}\right)$decay with $\mathcal{O}_{L ; L}$ as a function of invariant mass squared of lepton.

diamond in Fig. 1 1 represents this indirect estimation. There is no significant difference between direct and indirect results in the current statistics. However, $40 \%$ excess for the central value from indirect method is quite similar to the JLQCD result [3]. Similar of result is obtained also for $\left\langle\pi^{0}\left|\mathcal{O}_{R ; L}^{\not B}\right| p\right\rangle$.

The above results need be renormalized for the value in the $\overline{\mathrm{MS}}$ scheme in continuum theory. We expect these operators are multiplicatively renormalized as the chiral symmetry breaking is small $\left(m_{\text {res }} \sim 1.5 \mathrm{MeV}\right)$. The renormalization factor for DBW2 gauge action is available for one-loop perturbation theory [10] with $L_{s}$ treated as infinity. In Table 2 we list the values of renormalization factors for the nucleon decay by perturbation theory with two different mean field (MF) improvement schemes. The MF factor $u$ is either $P^{1 / 4}$ or $1 / 8 K_{c}$, where $P$ stands for plaquette, $K_{c}$ 
Table 2

Renormalization factors for the axial vector current and the nucleon decay three-quark operator in $\overline{\mathrm{MS}}$ with NDR. Scale $\mu=1 / a$ is chosen for nucleon decay.

\begin{tabular}{c|cc|c}
\hline \multirow{2}{*}{ operator } & \multicolumn{2}{|c|}{ perturbative 10} & non \\
& plaq & $K_{c}$ & perturbative \\
\hline $\bar{q} \gamma_{5} \gamma_{\mu} t^{a} q$ & 0.976 & 0.769 & $0.7776(5)$ \\
$\mathcal{O}_{L ; R / L}^{\not B}$ & 1.009 & 0.713 & - \\
\hline
\end{tabular}

the critical hopping parameter of the four dimensional Wilson fermion. We also list the renormalization factor for the axial vector current, for which we know the non-perturbative value at the parameter point of (b) in Table 1 with the method using the conserved current [6]. For the axial vector current renormalization, the mean field improvement with $K_{c}$ is in better agreement with the non-perturbative one, while the other is $30 \%$ larger. This difference is considered as the systematic error of the perturbative estimate. The difference arises mainly from the quark wave function renormalization and the MF factor. Thus, this problem does not apply to quantities defined by a particular ratio in which those factors cancel out. $B_{K}$ is one of those cases.

In Table 3 we list the result of $\alpha$ and $\beta$ of ours and by JLQCD. The operators are renormalized at $\mu=1 / a$ with $\overline{\mathrm{MS}}$ for both results. Renormalization factor with $K_{c}$ is employed for our calculation. Our values of $\alpha$ and $\beta$ in $\left[\mathrm{GeV}^{3}\right]$ obtained by setting the scale with the $\rho$ mass input, are quite different from those with Wilson fermion by JLQCD. The origin of the discrepancy could be, 1) discretization error, 2) systematic error of the MF perturbation, 3) difference of the renormalization scale. 2) is about $30 \%$ for DWF, 3)

\section{Table 3}

Chiral lagrangian parameter renormalized at $\mu=$ $1 / a$ in $\overline{\mathrm{MS}}$ in NDR. Scale from $\rho$ mass is used for results in $\left[\mathrm{GeV}^{3}\right]$. Quoted errors are statistical only.

\begin{tabular}{c|cc|cc}
\hline & \multicolumn{2}{|c|}{$\left[\mathrm{GeV}^{3}\right] \times 10^{-2}$} & & \\
& $|\alpha|$ & $|\beta|$ & $|\alpha| r_{0}^{3}$ & $|\beta| r_{0}^{3}$ \\
\hline This work & $0.6(1)$ & $0.7(1)$ & $0.15(3)$ & $0.18(4)$ \\
JLQCD[3] & $1.5(1)$ & $1.4(1)$ & $0.19(2)$ & $0.18(2)$ \\
\hline
\end{tabular}

is almost negligible. 1) is expected to be most severe as it is seen in the difference of the scale from $\rho$ mass for Wilson and DWF with Wilson gauge background at same $1 / g^{2}$ (see (c) and (d) in Table 1). Indeed, if we set the scale from different quantity $r_{0}$, which eliminates order $a$ error in the scale for Wilson fermion, the values are consistent.

\section{SUMMARY}

We calculated proton decay matrix elements with DBW2 gauge action and DWF. We did not find a significant difference in values obtained by direct and indirect methods within our current numerical precision. Further simulation with non-degenerate quark masses will reduce the error of direct calculation and make the extraction of nucleon to kaon decay amplitudes possible. Within the current systematic error of the perturbation theory the chiral lagrangian parameters for the nucleon decay calculated with DWF are consistent with those obtained with Wilson fermion by JLQCD if the scale is set by $r_{0}$. More stringent comparison will be done for those values after non-perturbative renormalization is performed.

\section{REFERENCES}

1. For summary and references, see [3].

2. M.B. Gavela et al., Nucl. Phys. B312 (1989) 269.

3. JLQCD, S. Aoki et al., Phys. Rev. D62 (2000) 014506, hep-lat/9911026.

4. RBC, K. Orginos, Nucl. Phys. Proc. Suppl. 106 (2002) 721, hep-lat/0110074.

5. RBC, Y. Aoki, Nucl. Phys. Proc. Suppl. 106 (2002) 245, hep-lat/0110143.

6. T. Blum et al., (2000), hep-lat/0007038.

7. ALPHA, M. Guagnelli, R. Sommer and H. Wittig, Nucl. Phys. B535 (1998) 389, heplat/9806005.

8. M. Claudson, M.B. Wise and L.J. Hall, Nucl. Phys. B195 (1982) 297.

9. S.Y. Hsueh et al., Phys. Rev. D38 (1988) 2056.

10. S. Aoki et al., (2002), hep-lat/0206013. 